\title{
The Effect and Management of Delayed Vasospasm after Aneurysmal Subarachnoid Hemorrhage
}

\author{
Nicholas W. C. DORSCH \\ Department of Neurosurgery, Westmead Hospital, Sydney, New South Wales, Australia
}

\begin{abstract}
Delayed cerebral vasospasm after aneurysm rupture is one of the major complications of subarachnoid hemorrhage. The purpose of this review was to determine the true incidence of vasospasm. All literature on cerebral aneurysms from 1960 onwards was reviewed, and the figures extracted from publications that mentioned vasospasm. Angiographic vasospasm, where patients were studied at the time of peak incidence, was reported in about two thirds of cases. Symptomatic vasospasm or delayed ischemia affects about one third. Untreated, nearly a third of those with ischemic deficits die and a similar proportion are left permanently disabled. Variations of Triple-H (hypervolemia, hypertension, hemodilution) therapy, used early after hemorrhage for prophylaxis of vasospasm, are associated with a decrease of nearly half in the incidence of delayed ischemia. When used as therapy outcome also appears better, with a reduction particularly in the death rate. Calcium antagonists have been widely used, especially nimodipine. In several controlled trials the incidence of delayed ischemia was significantly reduced. More importantly, the overall outcome of all subarachnoid hemorrhage patients was better with nimodipine prophylaxis. The 21-aminosteroid tirilazad mesylate has been the subject of several trials. In one the overall outcome of all patients was improved, but the effect was essentially in males only. Further studies with larger doses in females are being analyzed.
\end{abstract}

Key words: cerebral aneurysm, cerebral vasospasm, delayed ischemic deficit, Triple-H therapy, calcium antagonists, 21-aminosteroids

\section{Introduction}

Cerebral vasospasm following aneurysmal subarachnoid hemorrhage (SAH) is a major cause of disability. The problem of recurrent hemorrhage has been reduced by early operation. Since little can be done as yet to ameliorate the deleterious effects of the $\mathrm{SAH}$ itself, vasospasm is the major remaining problem requiring prevention or treatment.

Since the original description by Robertson ${ }^{101}$ of the pathological effects of ischemia after $\mathrm{SAH}$, and the first description of angiographic changes by Ecker and Riemenschneider, ${ }^{5}$ the importance of this complication has been recognized. However, information on the true incidence and effects of vasospasm has been sparse. Knowledge of these is important in the design of drug or other trials for the prophylaxis or treatment of vasospasm (for the calculation of patient numbers needed for the trial), in the assessment of results in individual centers, or in the prediction of possible complications in individual cases.

The actual cause of vasospasm is uncertain. The risk and severity of delayed spasm are undoubtedly related to the volume of subarachnoid blood seen on an early computed tomography scan. ${ }^{6}$ It is presumed that a breakdown product of the blood, the most favored at present being oxyhemoglobin, causes a cascade of chemical events within the arterial wall. This leads to muscular contraction and to structural changes that result in severe constriction of the involved arteries.

The exact magnitude of the effect of vasospasm is largely unknown. A review of the literature on aneurysms between 1960 and 1992, involving more than 1100 sources and 60,000 patients, has been carried out in order to clarify these questions. ${ }^{2-4)}$ In addition, changes in treatment that have taken place over the last two decades, following comprehensive reviews of treatment by Wilkins, ${ }^{12,13]}$ which discussed more than 80 different treatments that had been used in attempts to overcome this complication, are reviewed. 


\section{Natural History of Vasospasm}

\section{Incidence}

Vasospasm develops several days after SAH, peaking in severity usually about halfway through the second week. It is classically detected by cerebral angiography, and if angiograms to look for spasm could be done daily, a very high incidence would certainly be shown. The actual incidence in the reviewed literature, with data obtained from 216 references, was 13,129 cases of vasospasm in 30,389 reported patients, or $43.2 \%$.

The above figures included all reports that mentioned angiographic spasm, regardless of definition. In those reports in which it was stated that angiography was carried out around the end of the first week or in the second week after SAH, i.e. at the time of maximum incidence, the incidence was always higher, and averaged $67.0 \%$ (vasospasm in 1808 of 2699 patients). This latter figure is much more likely to approximate the true incidence.

Symptomatic vasospasm, or delayed ischemic deficit (DID), is considerably less common. There are several reasons for this: cerebral blood flow does not decrease until the spasm is severe and arterial diameter reduced by half or more (the increased velocity of flow measured by transcranial Doppler can be said to compensate for the reduced diameter); when blood flow is eventually reduced, the extraction of oxygen and nutrients from the blood is increased; and other factors such as blood pressure, intracranial pressure, and electrolyte imbalance play a part. Among 30,783 reported cases of SAH, DID occurred in 9882 or $32.1 \%$ (271 references).

\section{Effects}

The outcome of DID was mentioned in 105 reports. In a total of 3306 reported patients, 1004 $(30.4 \%)$ died, and permanent deficits were reported in 1126 cases (34.1\%). The remaining 1176 (35.6\%) eventually made a good recovery.

To summarize the natural history, angiographic vasospasm occurs in about two thirds of patients, and symptomatic spasm in one third. For those affected by DID, nearly one third die and a similar proportion are left disabled.

\section{Fluids and Hypertensive Therapy}

Since the first report by Kosnik and Hunt ${ }^{8)}$ of the use of induced hypertension to treat established DID, many variations of this type of treatment have been introduced. Some have used simply increased fluid loading, others hypertensive treatment with vasopressor drugs, and some the whole spectrum of hypervolemia, hypertension, and hemodilution, or Triple- $H$ therapy. ${ }^{91}$ The reported figures for the incidence or outcome of vasospasm with these various treatments are not greatly different, and in this study they have been combined.

When Triple- $H$ therapy or its variations were used for prophylaxis of vasospasm, that is, administered to all SAH patients as soon as possible after their hemorrhage ( 28 references), the incidence of DID in 2361 cases was 420 , or $17.8 \%$. This represents a considerable decrease compared with the incidence untreated, by nearly half.

For the treatment of established DID (67 reports), the outcome figures with Triple- $\mathrm{H}$ therapy were: death $325 / 1920=16.9 \%$, permanent deficits 549 or $28.6 \%$, and good outcomes in 1046 , or $54.5 \%$. This is again better than the figures without treatment, particularly where death rate is concerned. These treatments have never been submitted to any sort of controlled trial, but with such striking differences in such large numbers of cases, it is very likely that the differences are genuine.

\section{Calcium Antagonists}

Several different calcium antagonists have been used in the management of vasospasm. By far the most reported experience has been with the dihydropyridine analogue nimodipine, and most of the data presented here are concerned with that drug.

\section{Clinical trials}

A number of well-designed controlled trials of nimodipine have been reported. Combining these with other studies that used historical controls (total eight studies), very significant reductions in DID were seen - in 978 control patients the incidence was $310(31.7 \%)$, and in 702 who received nimodipine there was $22.4 \%$ (157 cases) DID $\left(\chi^{2}=17.3, \mathrm{p}\right.$ $<0.0001$ ).

In 10 more or less controlled studies, including four of the above, information was available on the outcome of patients. The death rate was $16.6 \%$ (192/ $1159)$ in controls and $11.8 \%(115 / 978)$ in treated cases $\left(\chi^{2}=9.6, p=0.0002\right)$; the incidence of all bad outcomes, death and permanent deficits combined (regardless of cause) was also considerably reduced, with $331 / 1166(28.4 \%)$ in control patients compared with $181 / 923(19.6 \%)$ with treatment $\left(\chi^{2}=21\right.$, p < 0.0001 ). A meta analysis by Tettenborn and Dycka ${ }^{11)}$ of seven controlled studies confirmed a highly significant improvement in outcome with nimodipine, and showed a reduction of $42 \%$ in the risk of a bad outcome. Recently, a more strict analysis of con- 
trolled studies has led to the conclusion that this drug is effective as part of the management of $\mathrm{SAH}^{11}$

\section{Prophylaxis}

Forty-seven references mentioned the use of nimodipine for the prevention of DID. Among 4128 patients DID occurred in 558, or $13.5 \%$. Although it has never been tested in a controlled way, it is possible that intravenous nimodipine is more effective than the oral preparation - the overall incidence of DID was $12.2 \%$ (418/3421) and $19.8 \%$ (140/707), respectively.

\section{Treatment}

In some reports of patients receiving prophylactic nimodipine, the outcome of those who developed DID and in whom the nimodipine was continued was given (31 references, 419 patients). 18\% were dead, $32 \%$ had permanent disability, and $50 \%$ made a good recovery.

In other reports, nimodipine was started de novo after the patients had already developed DID, i.e. it was used only for treatment. The 343 cases (six references) in this category include 141 of our own patients, in a period when nimodipine was only allowed to be used for treatment. Outcome for this group was apparently better, with 45 deaths $(13 \%)$, 68 permanent deficits (20\%), and $230(67 \%)$ making good recoveries.

\section{Other calcium antagonists}

Nicardipine has been used as prophylaxis in 1045 reported patients (12 references), with an incidence of DID of $24 \%$ (247 cases). When it was used as treatment (seven reports, 191 patients) death rate was $12 \%$, deficits $17 \%$, and good outcomes $71 \%$.

In 310 reported cases receiving flunarizine (three references) DID occurred in only $15(5 \%)$; the outcome of 13 of these was reported, with $11(85 \%)$ good recoveries. With diltiazem the reported incidence of DID (166 patients in four reports) was $32 \%$, and the outcome in treatment of DID included $46 / 68(68 \%)$ good. For verapamil, with only 34 cases in two reports, there was a $15 \%$ (five cases) incidence of DID.

\section{v. Side effects and complications}

Systemic hypotension is the most common problem encountered with nimodipine, and is more likely in previously hypertensive patients. In our own series, support with vasopressor drugs was needed in a number of cases, along with increased fluids.

Other complications have in general not been serious, and have recovered when the drug was stopped.
Such reversible problems, usually not clinically evident, include disturbances of liver function, hyperglycemia, and occasionally arterial hypoxemia.

One concern is that, if these drugs dilate cerebral arteries, they may increase the risk of rebleeding in patients with aneurysms. In fact, where this complication was mentioned, in four more or less controlled studies of nimodipine, there was slightly though not significantly less rebleeding in the treated group (54/448 or $12 \%)$ than in controls $(80 / 523$, $15 \%)$. Overall there were 130 recurrent hemorrhages in 1221 reported cases (11 references), or $11 \%$.

\section{Modified Steroids}

The 21-aminosteroid drug tirilazad mesylate, which is a potent inhibitor of iron-dependent lipid peroxidation, has been the subject of intensive clinical trials in head injury, ischemic stroke, and aneurysmal $\mathrm{SAH}$. The first large trial in SAH was carried out in Europe, Australia, and New Zealand, with all patients receiving intravenous nimodipine as well. The highest of the three dose levels tested, $6 \mathrm{mg} / \mathrm{kg} /$ day, showed a trend towards a reduced incidence of delayed ischemia. More importantly, the overall outcome was improved. Death rate was significantly reduced, and there was an increase in the proportion making a good recovery. ${ }^{7}$ Analysis of subgroups showed that the improvement in outcome affected male patients only. Interestingly, the improved outcome was seen also in males in clinical grades 4 and 5. In a confirmatory study in centers in North America, with oral nimodipine as background treatment, a similar effect was seen, with improved outcome in males only.

Further testing of the drug showed pharmacokinetic differences between the sexes, so that after the same dose, there was a much lower blood level of tirilazad and its main metabolite in females than in males. As a consequence, two more clinical trials have been carried out in females only, using a higher dose of the drug. These trials have been completed but their results are still being analyzed.

\section{Other Techniques}

A variety of other drugs and techniques have been used, and some are still under active investigation. They have been summarized more fully elsewhere. ${ }^{2 j}$

Variants of cisternal washout or irrigation have been used in many centers. At its simplest this consists of an external ventricular or cisternal drain. Others have used ventriculo-cisternal or lumbar perfusion. In some centers drugs are instilled, usually streptokinase or urokinase, and more recently tissue 
plasminogen activator, designed to dissolve cisternal clots and thus reduce the risk of vasospasm. Controlled studies of cisternal tissue plasminogen activator have been completed but the results are not yet published. In early reports the average incidence of DID was about $10 \% .{ }^{2)}$

Transluminal angioplasty has found a place mainly in the treatment of established symptomatic vasospasm. Impressive results have been obtained, particularly when one considers that in many of these patients other methods have already been tried and have failed. The average death rate in early studies was $20 \%$. Balloon dilatation is the preferred technique. Where this is not possible because of lack of expertise or equipment, or for spasm in very distal arteries, "chemical angioplasty," with selective catheterization and injection of papaverine or a calcium antagonist, has been used instead of or in combination with mechanical angioplasty.

\section{Discussion}

Vasospasm and delayed ischemia are important complications of aneurysmal SAH. Untreated, more than $20 \%$ of patients die or are permanently disabled because of DID. Calculating from the figures available, the use of calcium antagonists for prophylaxis, combined with appropriate fluid management and continued as treatment when necessary, should reduce the incidence of permanent problems due to vasospasm by half or more. The aminosteroid tirilazad mesylate used in combination with the above should, at least in males, give a further reduction.

It is well recognized that delayed vasospasm is a complex process involving a cascade of chemical steps. It would seem logical that this process should be attacked at multiple points in the chain, with different drugs and techniques for different phases. An example would be early operation followed by cisternal irrigation with a clot dissolver, fluids with or without pressor drugs to maintain cerebral perfusion, a calcium antagonist and aminosteroid for vasospasm prophylaxis and neuroprotection, and angioplasty at a later stage if it becomes necessary.

It is difficult to be sure exactly how the various drugs work. Multiple possible mechanisms of action have been postulated or are possible for calcium antagonists. Seven most likely mechanisms include: prevention or reversal of spasm, opening up of collaterals, protection of neurons against ischemia, intracellular actions in vessel walls or neurons, rheological effects, an anti-mitogenic effect, and improved management of fluids. ${ }^{3)}$ Similarly, the improved outcome in the published study of tirilazad mesylate ${ }^{7)}$ was against a background of no improvement in angiographic vasospasm and a (strong) trend towards a reduction in symptomatic spasm. Possibly, several different mechanisms may be in operation at different stages of the course of vasospasm, or in different patients. However, there is little doubt that many of these drugs are effective in preventing vasospasm or minimizing its effects, and in improving outcome after aneurysmal SAH.

\section{References}

1) Barker FG II, Ogilvy CS: Efficacy of prophylactic nimodipine for delayed ischemic deficit after subarachnoid hemorrhage: a metaanalysis. J Neurosurg 84: 405-414, 1996

2) Dorsch NWC: A review of cerebral vasospasm in aneurysmal subarachnoid haemorrhage. II. Management. Journal of Clinical Neuroscience 1: 78-92, 1994

3) Dorsch NWC: A review of cerebral vasospasm in aneurysmal subarachnoid haemorrhage. III. Mechanisms of action of calcium antagonists. Journal of Clinical Neuroscience 1: 151-160, 1994

4) Dorsch NWC, King MT: A review of cerebral vasospasm in aneurysmal subarachnoid haemorrhage. I. Incidence and effects. Journal of Clinical Neuroscience 1: 19-26, 1994

5) Ecker A, Riemenschneider PA: Arteriographic demonstration of spasm of the intracranial arteries. With special reference to saccular arterial aneurysms. J Neurosurg 8: 660-667, 1951

6) Fisher CM, Kistler JP, Davis TM: Relation of cerebral vasospasm to subarachnoid hemorrhage visualized by computerized tomographic scanning. Neurosurgery 6: 1-9, 1980

7) Kassell NF, Haley EC Jr, Apperson-Hansen C, Alves WM, the participants: Randomized, double-blind, vehicle-controlled trial of tirilazad mesylate in patients with aneurysmal subarachnoid hemorrhage. $J$ Neurosurg 84: 221-228, 1996

8) Kosnik EJ, Hunt WE: Postoperative hypertension in the management of patients with intracranial arterial aneurysms. J Neurosurg 45: 148-154, 1976

9) Origitano TC, Wascher TM, Reichman OH, Anderson DE: Sustained increased cerebral blood flow with prophylactic hypertensive hypervolemic hemodilution ("Triple- $\mathrm{H}$ " therapy) after subarachnoid hemorrhage. Neurosurgery 27: 729740,1990

10) Robertson EG: Cerebral lesions due to intracranial aneurysms. Brain 72: 150-185, 1949

11) Tettenborn D, Dycka T: Prevention and treatment of delayed ischemic dysfunction in patients with aneurysmal subarachnoid hemorrhage. Stroke 21 (Suppl IV): IV85-IV89, 1990

12) Wilkins RH: Attempted prevention or treatment of in tracranial arterial spasm: a survey. Neurosurgery 6: 198-210, 1980

13) Wilkins RH: Attempts at prevention or treatment of 
intracranial arterial spasm: an update. Neurosurgery 18: $808-825,1986$
Address reprint requests to: N. W. C. Dorsch, M.D., Department of Surgery, Westmead Hospital, Westmead, NSW 2145, Sydney, Australia. 